peptic ulcer disease (PUD) and its complications among hospitalized patients. METHODS/STUDY POPULATION: We performed casecontrol studies with records from the NHANES III $(n=4,556)$ and HCUP-NIS 2014 ( $n=4,555,029)$, and respectively identified subjects with seropositivity for $\mathrm{H}$ pylori and clinical PUD, and their cannabis usage status. In the NHANES III, we estimated the adjusted prevalence rate ratio (aPRR) of having HPI with cannabis use, using generalized estimating equations. In the NIS, we propensity-matched cannabis users to non-users in ratio $1: 1(68,073: 68,073)$ and measured the aPRR of having PUD and its complications (SAS 9.4). RESULTS/ANTICIPATED RESULTS: In NHANES III, associated with decreased HPI seropositivity were cannabis ever-users (aPRR: $0.79[0.66-0.95])$, greater than 10 times lifetime usage $(0.65[0.5-0.84])$ and recent 31 -day usage $(0.67[0.48-0.98])$, compared to never usage. In the HCUP-NIS, cannabis users had decreased risk for total PUD (aPRR: 0.74[0.61-0.89]), duodenal PUD (0.48[0.35-0.60]) and PUD complications including hemorrhage (0.58[0.37-0.90]), perforation (0.66[0.51-0.87]), but not obstruction (1.75[0.51-5.98]). DISCUSSION/SIGNIFICANCE OF IMPACT: Cannabis usage is related to a reduced likelihood of having HPI in the community and also mitigate against having complicated presentations to the hospital. More translational studies are needed to illuminate the details of this relationship, given the high worldwide prevalence of both cannabis use and HPI.

\section{Cardiac Replacement Fibrosis in Cancer Treatment Related Cardiotoxicity}

Chetan Shenoy ${ }^{1}$ and Kalpit Modi

${ }^{1}$ University of Minnesota CTSI

OBJECTIVES/SPECIFIC AIMS: Our goals were to understand the pattern, location, and extent of cardiac replacement fibrosis seen as late gadolinium enhancement on cardiovascular magnetic resonance imaging in a large cohort of cancer patients treated with anthracyclines and/or trastuzumab. METHODS/STUDY POPULATION: We performed a retrospective cohort study of consecutive adult cancer patients treated with anthracyclines and/or trastuzumab from 2004 through 2017. CMRs were analyzed for the presence, location, and pattern of LGE. RESULTS/ ANTICIPATED RESULTS: Of 238 patients, 220/(92.4\%) had no LGE. Among the 18/(7.6\%) patients with LGE, 13/(72.2\%) were ischemic in pattern (myocardial infarctions); 10 of these had known coronary artery disease (CAD). Of $5 /(27.8 \%)$ patients with nonischemic LGE, the etiologies were known for 4 - myocarditis, cardiac sarcoidosis, eosinophilic myocarditis, and acute myocardial calcification. Only $4 /(1.7 \%)$ patients had unexpected LGE, of which 3 were unrecognized myocardial infarctions. DISCUSSION/ SIGNIFICANCE OF IMPACT: The assessment of fibrosis helps to diagnose the cause of LVSD in cancer patients treated with potentially cardiotoxic medications. This is necessary because currently, the cause of LVSD in cancer patients cannot be established conclusively even though the cause is closely linked to patient outcomes. Our results demonstrate that cancer treatment-related LVSD is not associated with fibrosis. A minority of cancer patients with LVSD have fibrosis related to other reasons, most commonly CAD. Identification of the correct cause of LVSD in cancer patients treated with cardiotoxic medications allows for appropriate treatment. This, in turn, could improve patient outcomes.
Catatonia, Delirium and Coma: Implications for Mortality Jo Ellen Wilson ${ }^{1}$, Sarasota Mihalko, Stephan Heckers, Pratik P. Pandharipande, Timothy D. Girard, Ahra Kim, Simon Vandekar, Rameela Chandrasekhar, Andrew Francis, Robert S. Dittus and Eugene "Wes" Ely

${ }^{1}$ Vanderbilt University Medical Center

OBJECTIVES/SPECIFIC AIMS: Delirium, a form of acute brain dysfunction, characterized by changes in attention and alertness, is a known independent predictor of mortality in the Intensive Care Unit (ICU). We sought to understand whether catatonia, a more recently recognized form of acute brain dysfunction, is associated with increased 30-day mortality in critically ill older adults. METHODS/STUDY POPULATION: We prospectively enrolled critically ill patients at a single institution who were on a ventilator or in shock and evaluated them daily for delirium using the Confusion Assessment for the ICU and for catatonia using the Bush Francis Catatonia Rating Scale. Coma, was defined as a Richmond Agitation Scale score of -4 or -5 . We used the Cox Proportional Hazards model predicting 30-day mortality after adjusting for delirium, coma and catatonia status. RESULTS/ ANTICIPATED RESULTS: We enrolled 335 medical, surgical or trauma critically ill patients with 1103 matched delirium and catatonia assessments. Median age was 58 years (IQR: 48 - 67). Main indications for admission to the ICU included: airway disease or protection $(32 \% ; \mathrm{N}=100)$ or sepsis and/or shock $(25 \% ; \mathrm{N}=79$. In the unadjusted analysis, regardless of the presence of catatonia, non-delirious individuals have the highest median survival times, while delirious patients have the lowest median survival time. Comparing the absence and presence of catatonia, the presence of catatonia worsens survival (Figure 1). In a time-dependent Cox model, comparing non-delirious individuals, holding catatonia status constant, delirious individuals have 1.72 times the hazards of death (IQR: 1.321, 2.231) while those with coma have 5.48 times the hazards of death (IQR: 4.298, 6.984). For DSM-5 catatonia scores, a 1-unit increase in the score is associated with 1.18 times the hazards of in-hospital mortality. Comparing two individuals with the same delirium status, an individual with a DSM-5 catatonia score of 0 (no catatonia) will have 1.178 times the hazard of death (IQR: 1.086, 1.278), while an individual with a score of 3 catatonia items (catatonia) present will have 1.63 times the hazard of death. DISCUSSION/SIGNIFICANCE OF IMPACT: Non-delirious individuals have the highest median survival times, while those who are comatose have the lowest median survival times after a critical illness, holding catatonia status constant. Comparing the absence and presence of catatonia, the presence of catatonia seems to worsen survival. Those individual who are both comatose and catatonic have the lowest median survival time.

Colonization of Pregnant Women with Group B streptococcus in Latin America and Infant Outcomes

Elena HogenEsch ${ }^{1}$, Lisa Haddad, Inci Yildirim and Saad B Omer ${ }^{1}$ Emory University

OBJECTIVES/SPECIFIC AIMS: The primary objective of this study is to determine the prevalence of maternal GBS colonization and demographic risk factors associated with maternal GBS colonization 